\title{
TWO FUNCTION-SPACE TOPOLOGIES
}

\author{
G. A. EDGAR
}

\begin{abstract}
Two topologies for the set of all continuous functions with compact support are shown to be distinct. One of the topologies is the usual inductive limit topology, and the other is a topology claimed by $\mathrm{G}$. Choquet to coincide with it.
\end{abstract}

One approach to the Lebesgue integral [2a, Chapter III, §1] uses a topology defined as follows. Let $E$ be a locally compact Hausdorff space, and let $\mathscr{K}$ be the set of all real-valued continuous functions with compact support on $E$. For each compact set $K \subseteq E$, let $\mathscr{K}(K)$ be the set of functions in $\mathscr{K}$ with support included in $K$; give $\mathscr{K}(K)$ the topology of uniform convergence. Topologize $\mathscr{K}$ with the finest locally convex topology such that the inclusions $\mathscr{K}(K) \hookrightarrow \mathscr{K}$ are all continuous. G. Choquet [3, p. 301] omits the words "locally convex", but claims [3, Proposition 16.4, p. 302] that the two topologies are the same. We deny that claim here.

THEOREM. If $E$ is the real line, then the two topologies defined above are distinct.

Proof. Let $\tau$ denote the locally convex topology and $\rho$ the Choquet topology. For $f \in \mathscr{K}$, write $\|f\|=\sup _{x}|f(x)|$. For $n=1,2, \cdots$, let $\mathscr{K}_{n}=$ $\mathscr{K}([-n, n])$. Thus a set $\mathscr{U} \subseteq \mathscr{K}$ is $\rho$-open if and only if $\mathscr{U} \cap \mathscr{K}_{n}$ is open in $\mathscr{K}_{n}$ for every $n$; and a convex set $\mathscr{U} \subseteq \mathscr{K}$ is $\tau$-open if and only if $\mathscr{U} \cap \mathscr{K}_{n}$ is open in $\mathscr{K}_{n}$ for every $n$.

For integers $n, m \geqq 2$, let $f_{n m} \in \mathscr{K}$ be the piecewise linear function with vertices $(0,0),(1 / m, 1 / n),(1,1 / m),(n, 0)$. Let $\mathscr{F}_{n}=\left\{f_{n m}: m=2,3, \cdots\right\}$ and let $\mathscr{F}=\bigcup_{n=2}^{\infty} \mathscr{F}_{n}$. We first show that $\mathscr{F}$ is $\rho$-closed. No infinite subset of $\mathscr{F}_{n}$ is equicontinuous at 0 , so no sequence in $\mathscr{F}_{n}$ converges uniformly to a function not in $\mathscr{F}_{n}$. Therefore $\mathscr{F}_{n}$ is closed in $\mathscr{K}_{n}$. But then $\mathscr{F} \cap$ $\mathscr{K}_{n}=\bigcup_{i=2}^{n} \mathscr{F}_{i}$ is closed in $\mathscr{K}_{n}$. Hence $\mathscr{F}$ is $\rho$-closed.

We next show that the function 0 is a $\tau$-accumulation point of the set $\mathscr{F}$. Let $\mathscr{U}$ be an arbitrary $\tau$-neighborhood of 0 . By local convexity, there

Received by the editors November 29, 1972.

AMS (MOS) subject classifications (1970). Primary 46A05; Secondary 28A30, 46E10, 54A10, 54G20.

Key words and phrases. Locally convex topology, inductive limit topological vector space, compactly supported function, Radon measure, Lebesgue integral.

(c) American Mathematical Society 1973 
is a convex $\tau$-neighborhood $\mathscr{V}$ of 0 such that $\mathscr{V} \subseteq \mathscr{U}$. Since $\mathscr{V} \cap \mathscr{K}_{1}$ is a neighborhood of 0 in $\mathscr{K}_{1}$, there is a positive integer $n$ such that

$$
\mathscr{V} \cap \mathscr{K}_{1} \supseteq\left\{f \in \mathscr{K}_{1}:\|f\| \leqq 2 / n\right\} .
$$

Since $\mathscr{V} \cap \mathscr{K}_{n}$ is a neighborhood of 0 in $\mathscr{K}_{n}$, there is a positive integer $m$ such that

$$
\mathscr{V} \cap \mathscr{K}_{n} \supseteq\left\{f \in \mathscr{K}_{n}:\|f\| \leqq 2 / m\right\} \text {. }
$$

Now if $g \in \mathscr{K}$ is the piecewise linear function with vertices $(0,0),(1 / m$, $1 / n),(1,0)$, then $2 g \in \mathscr{V} \cap \mathscr{K}_{1}$ and $2\left(f_{n m}-g\right) \in \mathscr{V} \cap \mathscr{K}_{n}$, so that $f_{n m} \in$ $\mathscr{V} \subseteq \mathscr{U}$. Therefore 0 belongs to the $\tau$-closure of $\mathscr{F}$.

Thus $\mathscr{F}$ is $\rho$-closed, but not $\tau$-closed, so the two topologies are distinct.

REMARKS. (i) Another characterization of the topology $\tau$ is given in [2, Chapter III, §2, exercise 2a, p. 64]. (ii) By [1, Chapter II, §2, exercise 10, p. 68], $\tau$ is the finest topology for $\mathscr{K}$ such that addition is continuous and the inclusions $\mathscr{K}(K) \hookrightarrow \mathscr{K}$ are all continuous. Therefore, addition is not $\rho$-continuous. (iii) The above proof can be adapted to prove the same result for any $E$ which is $\sigma$-compact, noncompact, and has a nonisolated $G_{\delta}$-point. However, if $E$ is, for example, a countable discrete space, then it can be shown that $\tau=\rho$.

I wish to thank S. L. Portnoy for discussions on this topic.

\section{REFERENCES}

1. N. Bourbaki, Éléments de mathématique. Fasc. XV. Livre V: Espaces vectoriels topologiques, Actualités Sci. Indust., no. 1189, Hermann, Paris, 1953. MR 14, 880.

2. $\_$Éléments de mathématique. Fasc. XIII. Livre VI: Intégration, Actualités Sci. Indust., no. 1175, Hermann, Paris, 1952. MR 14, 960.

2a. ——, Ibid., 2nd ed., 1965. MR 36 \#2763.

3. G. Choquet, Lectures in analysis. Vol. I, Benjamin, New York, 1969. MR 40 \#3252.

Department of Mathematics, Harvard University, Cambridge, Massachusetts 02138 